\title{
NOTE ON GEOMETRY OF FOUR DIMENSIONS.
}

BY PROFESSOR E. O. LOVETT.

(Read before the American Mathematical Society, April 28, 1900.)

1. Speculations relative to the geometry of $n$ dimensional space have followed several fairly well-defined trends which not infrequently cross one another : $1^{\circ}$ A direct extension of the Cartesian geometry, which extension is to be regarded as nothing more than a convenient form of phraseology ; in this form did $n$ dimensional space spring forth from the minds of Grassmann, Cayley, Gauss, and Cauchy, and the idea was likely familiar to Euler and Lagrange. $2^{\circ}$ The transformation of the ordinary visualizable spaces of two and three dimensions into manifoldnesses of higher or lower dimensions by introducing space elements other than the point or its dual element; for example, the line geometry of Plücker, the sphere geometry of Lie, the five dimensional manifoldness of all conics in the plane as an auxiliary to Ball's theory of screws; this category becomes more concrete perhaps than any other. $3^{\circ}$ The absolute geometry of space; here would appear the celebrated dissertation of Riemann, the well-known memoirs of Helmholtz and Lie and the elaborate treatise of Veronese. $4^{\circ}$ The extension of the methods of ordinary differential geometry to spaces of many dimensions; to this class belong the works of Christoffel, Beltrami, Bianchi, Cesàro, and Ricci, and the quite recent contributions of Darboux and his pupils. $5^{\circ}$ The direct extension of the concepts and problems of metrical and projective geometry of ordinary space, as exemplified in the memoirs of Jordan, d'Ovidio, and Veronese. $6^{\circ}$ The theory of birational correspondences between $n$ dimensional aggregates as studied by Noether, Kantor, and Brill. $7^{\circ}$ The descriptive geometry of space of $n$ dimensions as begun in the papers of Veronese, Stringham, Schlegel, and Segre. $8^{\circ}$ The kinematics of higher spaces as developed by Jordan, Clifford, and Beltrami. $9^{\circ}$ The interpretation of $n$ dimensional geometry in the light of the theory of groups as exhibited by Lie, Klein, and Poincaré.

2. It is proposed here to make an expository contribution to the ninth and fourth of the above categories, constructing ordinary four dimensional space by the method of Lie's theory of continuous groups, and studying curves of triple 
curvature by the intrinsic analysis developed by Cesàro in his Lezioni di geometria intrinseca.

3. With regard to the space it is assumed:

$1^{\circ}$ That it is a four dimensional manifoldness, $i . e$., that four independent data are necessary and sufficient to determine the position of an element of the manifoldness; these four independent things are called the coördinates of the element.

$2^{\circ}$ That a figure of the manifoldness possesses ten degrees of freedom within the manifoldness; $i$. e., that ten independent data are necessary and sufficient to render a rigid body fixed in position; the latter ten independent things are called the parameters of the figure.

For convenience let the element be called a point, and its coördinates be designated by $x, y, z, t$. Consider any figure containing this point and let its parameters be $\alpha_{1}, \alpha_{2}, \cdots, \alpha_{10}$. Let the figure assume a new position and call $x^{\prime}, y^{\prime}, z^{\prime}, t^{\prime}$ the coördinates of the new position of $(x, y, z, t)$. Then

$x^{\prime}=\xi\left(x, y, z, t, \alpha_{1}, \alpha_{2}, \cdots, \alpha_{10}\right), \quad y^{\prime}=\eta\left(x, y, z, t, \alpha_{1}, \alpha_{2}, \cdots, \alpha_{10}\right)$, $z^{\prime}=\zeta\left(x, y, z, t, \alpha_{1}, \alpha_{2}, \ldots, \alpha_{10}\right), \quad t^{\prime}=\tau\left(x, y, z, t, \alpha_{1}, \alpha_{2}, \cdots, \alpha_{10}\right)$.

The operation changing $x, y, z, t$ into $\xi, \eta, \zeta, \tau$ represents one of the motions of a four dimensional figure, and the ensemble of all these operations constitutes a continuous group with ten parameters. The identical transformation ought to appear among these operations. There must then be a system of the parameters $\alpha_{1}, \alpha_{2}, \cdots, \alpha_{10}$ such that

$$
\xi=x, \quad \eta=y, \quad \zeta=z, \quad \tau=t .
$$

It is legitimate to assume the preceding system of values to be

$$
\alpha_{1}=\alpha_{2}=\cdots=\alpha_{10}=0 .
$$

An infinitesimal transformation of the group is one whose parameters differ by infinitesimals from those giving the identical transformation; in this case the infinitesimal transformation is obtained by assigning infinitesimal values to the parameters themselves ; $i$. e., by such a transformation $x, y, z, t$ are changed to

$$
x+\sum_{1}^{10} \alpha_{i} \frac{\partial \xi}{\partial \alpha_{i}}, \quad y+\sum_{1}^{10} \alpha_{i} \frac{\partial \eta}{\partial \alpha_{i}}, \quad z+\sum_{1}^{10} \alpha_{i} \frac{\partial \xi}{\partial \alpha_{i}}, \quad t+\sum_{1}^{10} \alpha_{i} \frac{\partial \tau}{\partial \alpha_{i}},
$$

in the partial derivatives of which the $\alpha$ 's should be put equal to zero. 
Writing for short

$$
p \equiv \frac{\partial f}{\partial x}, \quad q \equiv \frac{\partial f}{\partial y}, \quad r \equiv \frac{\partial f}{\partial z}, \quad s \equiv \frac{\partial f}{\partial t},
$$

Lie's symbol for the above infinitesimal transformation is

Putting

$$
I \equiv p \sum_{1}^{10} \alpha_{i} \frac{\partial \xi}{\partial \alpha_{i}}+q \sum_{1}^{10} \alpha_{i} \frac{\partial \eta}{\partial \alpha_{i}}+r \sum_{1}^{10} \alpha_{i} \frac{\partial \zeta}{\partial \alpha_{i}}+s \sum_{1}^{10} \alpha_{i} \frac{\partial \tau}{\partial \alpha_{i}} .
$$

$$
J_{i} \equiv p \frac{\partial \xi}{\partial \alpha_{i}}+q \frac{\partial \eta}{\partial \alpha_{i}}+r \frac{\partial \zeta}{\partial \alpha_{i}}+s \frac{\partial \tau}{\partial \alpha_{i}}, \quad(i=1,2, \cdots, 10)
$$

any infinitesimal transformation is written

$$
I \equiv \sum_{1}^{10} \alpha_{i} J_{i}
$$

By a fundamental theorem of Lie, if we put

then

$$
\left(J_{i}, J_{s}\right) \equiv \frac{\partial J_{i}}{\partial p} \frac{\partial J_{j}}{\partial x}-\frac{\partial J_{i}}{\partial x} \frac{\partial J_{j}}{\partial p}+\cdots+\frac{\partial J_{i}}{\partial s} \frac{\partial J_{j}}{\partial t}-\frac{\partial J_{i}}{\partial t} \frac{\partial J_{j}}{\partial s}
$$

$$
\left(J_{i}, J_{j}\right)=\sum_{k=1}^{k=10} \lambda_{k} \lambda_{k}^{(i, j)}, \quad(i=1, \cdots, 10 ; j=1, \cdots, 10)
$$

where the $\lambda$ 's are constants. There are forty-five of these equations, but the four hundred and fifty $\lambda$ 's are not wholly arbitrary, since the following one hundred and twenty identities of Jacobi must hold

$$
\begin{gathered}
\left(J_{i},\left(J_{j}, J_{k}\right)\right)+\left(J_{j},\left(J_{k}, J_{i}\right)\right)+\left(J_{k},\left(J_{i}, J_{j}\right)\right)=0, \\
(i, j, k=1,2, \cdots, 10) .
\end{gathered}
$$

Every set of $J$ 's satisfying (1) and (2) reveals a space whose independent infinitesimal motions are represented by the infinitesimal operations of the set. Those functions of the elements that are invariant under these transformations will characterize the geometry of the space.

4. It may be verified without difficulty that the following forms for the fundamental transformations $J_{1}, J_{2}, \cdots, J_{10}$, which are functions of $x, y, z, t, p, q, r, s$, namely

$$
p, q, r, s, \quad \begin{aligned}
x q-y p, \quad y r-z q, \quad x s-t p \\
z p-x r, \quad t r-z s, \quad y s-t q,
\end{aligned}
$$

satisfy the conditions (1) and (2). 
Let $(x, y, z, t)$ and $\left(x^{\prime}, y^{\prime}, z^{\prime}, t^{\prime}\right)$ be any two points, and $\left(x_{1}, y_{1}, z_{1}, t_{1}\right),\left(x_{1}^{\prime}, y_{1}^{\prime}, z_{1}^{\prime}, t_{1}^{\prime}\right)$ be their positions after they are subjected to the transformation

$$
I \equiv \sum_{1}^{10} \alpha_{i} J_{i}
$$

where the $J_{i}^{\prime}$ s have the value (3).

Let $\varphi\left(x, y, z, t, x^{\prime}, y^{\prime}, z^{\prime}, t^{\prime}\right)$ be a function which is absolutely invariant under this operation ; then must

$$
\varphi\left(x_{1}, y_{1}, z_{1}, t_{1}, x_{1}^{\prime}, y_{1}^{\prime}, z_{1}^{\prime}, t_{1}^{\prime}\right)=\varphi\left(x, y, z, t, x^{\prime}, y^{\prime}, z^{\prime}, t^{\prime}\right),
$$

that is

$$
I \varphi \equiv 0
$$

for all values of the $\alpha_{i}^{\prime}$ 's hence equating to zero the coefficients of the $\alpha_{i}^{\prime} \mathrm{s}$ in $I \varphi$ we have the following system of linear partial differential equations for the function $\varphi$ :

$$
\begin{gathered}
\frac{\partial \varphi}{\partial x}+\frac{\partial \varphi}{\partial x^{\prime}}=0, \quad \frac{\partial \varphi}{\partial y}+\frac{\partial \varphi}{\partial y^{\prime}}=0, \quad \frac{\partial \varphi}{\partial z}+\frac{\partial \varphi}{\partial z^{\prime}}=0 \\
\frac{\partial \varphi}{\partial t}+\frac{\partial \varphi}{\partial t^{\prime}}=0 \\
x \frac{\partial \varphi}{\partial y}-y \frac{\partial \varphi}{\partial x}+x^{\prime} \frac{\partial \varphi}{\partial y^{\prime}}-y^{\prime} \frac{\partial \varphi}{\partial x^{\prime}}=0, \quad y \frac{\partial \varphi}{\partial z}-z \frac{\partial \varphi}{\partial y}+y^{\prime} \frac{\partial \varphi}{\partial z^{\prime}}-z^{\prime} \frac{\partial \varphi}{\partial y^{\prime}}=0 \\
x \frac{\partial \varphi}{\partial t}-t \frac{\partial \varphi}{\partial x}+x^{\prime} \frac{\partial \varphi}{\partial t^{\prime}}-t^{\prime} \frac{\partial \varphi}{\partial x^{\prime}}=0, \\
z \frac{\partial \varphi}{\partial x}-x \frac{\partial \varphi}{\partial z}+z^{\prime} \frac{\partial \varphi}{\partial x^{\prime}}-x^{\prime} \frac{\partial \varphi}{\partial z^{\prime}}=0, \quad t^{\frac{\partial \varphi}{\partial z}}-z \frac{\partial \varphi}{\partial t}+t^{\prime} \frac{\partial \varphi}{\partial z^{\prime}}-z^{\prime} \frac{\partial \varphi}{\partial t^{\prime}}=0 \\
y \frac{\partial \varphi}{\partial t}-t \frac{\partial \varphi}{\partial y}+y^{\prime} \frac{\partial \varphi}{\partial t^{\prime}}-t^{\prime} \frac{\partial \varphi}{\partial y^{\prime}}=0 .
\end{gathered}
$$

This system of ten equations in eight variables should not in general possess a solution. However the equations are not all independent. If the first seven be multiplied respectively by the following functions

$$
0, \quad 0, \quad \frac{x y^{\prime}-x^{\prime} y}{y-y^{\prime}}, \quad 0, \frac{z^{\prime} t-z t^{\prime}}{y t^{\prime}-y^{\prime} t}, \frac{x^{\prime}-x}{y-y^{\prime}}, \frac{y^{\prime} z-y z^{\prime}}{y t^{\prime}-y^{\prime} t}
$$

and the results be added, the eighth equation of the system is obtained; similarly, if the multipliers be 


$$
0, \quad 0, \frac{y t^{\prime}-y^{\prime} t}{y-y^{\prime}}, \frac{x^{\prime} t-x t^{\prime}}{x-x^{\prime}}, \quad 0, \quad \frac{t-t^{\prime}}{y-y^{\prime}}, \frac{z^{\prime}-z}{x-x^{\prime \prime}}
$$

the ninth equation results; and finally if the multipliers

$$
0, \quad 0, \quad 0, \quad \frac{x y^{\prime}-x^{\prime} y}{x-x^{\prime}}, \quad \frac{z t^{\prime}-z^{\prime} t}{x z^{\prime}-x^{\prime} z}, \frac{x t^{\prime}-x^{\prime} t}{x z^{\prime}-x^{\prime} z}, \frac{y-y^{\prime}}{x-x^{\prime}},
$$

be taken, the summation yields the tenth equation of the system.

The system composed of the first seven equations possesses at least one solution; that it has. no more is readily seen by noting the fact that not all seventh order determinants of the matrix

$$
\left\|\begin{array}{cccccccc}
0 & 0 & 0 & 0 & 1 & 0 & 0 & 0 \\
0 & 0 & 0 & 0 & 0 & 1 & 0 & 0 \\
0 & 0 & 0 & 0 & 0 & 0 & 1 & 0 \\
0 & 0 & 0 & 0 & 0 & 0 & 0 & 1 \\
y^{\prime}-y & x-x^{\prime} & 0 & 0 & -y^{\prime} & x^{\prime} & 0 & 0 \\
0 & z^{\prime}-z & y \frac{-}{0} y^{\prime} & 0 & 0 & -z^{\prime} & y^{\prime} & 0 \\
t^{\prime}-t & 0 & 0 & x-x^{\prime} & -t^{\prime} & 0 & 0 & x^{\prime}
\end{array}\right\|
$$

vanish; for example, the one formed by the last seven columns, whose value is written down at once as $\left(x-x^{\prime}\right)^{2}$ $\left(y-y^{\prime}\right)$.

The unique solution of this system appears by observing that the first four equations demand that the solution be a function only of

$$
X \equiv x-x^{\prime}, \quad Y \equiv y-y^{\prime}, \quad Z \equiv z-z^{\prime}, \quad T \equiv t-t^{\prime} ;
$$

the last three equations become

$X \frac{\partial \varphi}{\partial Y}-Y \frac{\partial \varphi}{\partial X}=0, \quad Y \frac{\partial \varphi}{\partial Z}-Z \frac{\partial \varphi}{\partial Y}=0, \quad X \frac{\partial \varphi}{\partial T}-T \frac{\partial \varphi}{\partial X}=0$

which require that $\varphi$ be of the form

$$
X^{2}+Y^{2}+Z^{2}+T^{2}
$$

that is, the function

$$
\sqrt{\left(x-x^{\prime}\right)^{2}+\left(y-y^{\prime}\right)^{2}+\left(z-z^{\prime}\right)^{2}+\left(t-t^{\prime}\right)^{2}}
$$

is an absolute invariant under the most general transformaton of the group (3). This function is said to define the distance between the two points $(x, y, z, t),\left(x^{\prime}, y^{\prime}, z^{\prime}, t^{\prime}\right)$. 
5. Consider now the linear element manifoldness

$$
\begin{aligned}
& x+l t+a=0, \\
& y+m t+b=0, \\
& z+n t+c=0 .
\end{aligned}
$$

It is proposed to find the variations experienced by $l, m, n$, $a, b, c$, under the operations (3). This determination is readily effected in the following manner :

The total variations of (5) give

$\delta(x+l t+a)=0, \quad \delta(y+m t+b)=0, \quad \delta(z+n t+c)=0$.

In (6) are substitued successively the increments assigned to $x, y, z, t$ by the transformations (3). A comparison of the resulting equations with the equations (5) gives equations for the variations of $l, m, n, a, b, c$. The results are tabulated below :

\begin{tabular}{ccccrcc} 
Transfor- & & \multicolumn{1}{c}{} & & & \\
mation. & $\delta l$ & $\delta m$ & $\delta n$ & $\delta a$ & $\delta b$ & $\delta c$ \\
$p$ & 0 & 0 & 0 & -1 & 0 & 0 \\
$q$ & 0 & 0 & 0 & 0 & -1 & 0 \\
$r$ & 0 & 0 & 0 & 0 & 0 & -1 \\
$s$ & 0 & 0 & 0 & $-l$ & $-m$ & $-n$ \\
$x q-y p$ & $-m$ & $l$ & 0 & $-b$ & $a$ & 0 \\
$y r-z g$ & 0 & $-n$ & $m$ & 0 & $-c$ & $b$ \\
$x s-t p$ & $l^{2}+1$ & $l m$ & $l n$ & $a l$ & $a m$ & $a n$ \\
$z p-x r$ & $n$ & 0 & $-l$ & $c$ & 0 & $-a$ \\
$t r-z s$ & $-l n$ & $-m n$ & $-\left(11+n^{2}\right)$ & $-c l$ & $-c m$ & $-c n$ \\
$y s-t q$ & $l m$ & $1+m^{2}$ & $m n$ & $b l$ & $b m$ & $b n$
\end{tabular}

Two linear manifoldnesses $(l, m, n, a, b, c),\left(l, m^{\prime}, n^{\prime}\right.$, $\left.a^{\prime}, b^{\prime}, c^{\prime}\right)$ possess invariants under the preceding transformations. These appear as solutions of the simultaneous system composed of the ten equations

$$
\begin{gathered}
\frac{\partial \varphi}{\partial l} \delta l+\frac{\partial \varphi}{\partial m} \delta m+\frac{\partial \varphi}{\partial n} \delta n+\frac{\partial \varphi}{\partial a} \delta a+\frac{\partial \varphi}{\partial b} \delta b+\frac{\partial \varphi}{\delta c} \delta c+\frac{\partial \varphi}{\delta l^{\prime}} \delta l^{\prime}+ \\
\cdots=0,
\end{gathered}
$$

formed by assigning successively to the variations the values given in the above table. This system has two independent solutions. These are analogous in form to the expression for the distance between two straight lines in ordinary space. There exists however another unique invariant of the two linear manifoldnesses, namely 


$$
\begin{gathered}
m \frac{\partial \varphi}{\partial l}-l \frac{\partial \varphi}{\partial m}+m^{\prime} \frac{\partial \varphi}{\partial n^{\prime}}-l^{\prime} \frac{\partial \varphi}{\partial m^{\prime}}=0 \\
n \frac{\partial \varphi}{\partial m}-m \frac{\partial \varphi}{\partial n}+n^{\prime} \frac{\partial \varphi}{\partial m^{\prime}}-m^{\prime} \frac{\partial \varphi}{\partial n^{\prime}}=0 \\
l \frac{\partial \varphi}{\partial n}-n \frac{\partial \varphi}{\partial l}+l^{\prime} \frac{\partial \varphi}{\partial n^{\prime}}-n^{\prime} \frac{\partial \varphi}{\partial l^{\prime}}=0 \\
\left(l^{2}+1\right) \frac{\partial \varphi}{\partial l}+l m \frac{\partial \varphi}{\partial m}+l n \frac{\partial \varphi}{\partial n}+\left(l^{2}+1\right) \frac{\partial \varphi}{\partial l^{\prime}} \\
+l^{\prime} m^{\prime} \frac{\partial \varphi}{\partial m^{\prime}}+l^{\prime} n^{\prime} \frac{\partial \varphi}{\partial n^{\prime}}=0 \\
l m \frac{\partial \varphi}{\partial l}+\left(m^{2}+1\right) \frac{\partial \varphi}{\partial m}+n m^{2} \frac{\partial \varphi}{\partial n}+l^{\prime} m^{\prime} \frac{\partial \varphi}{\partial l^{\prime}} \\
+\left(m^{\prime 2}+1\right) \frac{\partial \varphi}{\partial m^{\prime}}+m^{\prime} n^{\prime} \frac{\partial \varphi}{\partial n^{\prime}}=0, \\
l n \frac{\partial \varphi}{\partial l}+m n \frac{\partial \varphi}{\partial m}+\left(n^{2}+1\right) \frac{\partial \varphi}{\partial n}+l^{\prime} n^{\prime} \frac{\partial \varphi}{\partial l^{\prime}}+m^{\prime} n \frac{\partial \varphi}{\partial m^{\prime}} \\
+\left(n^{\prime 2}+1\right) \frac{\partial \varphi}{\partial n^{\prime}}=0,
\end{gathered}
$$

which possesses a solution since the determinant of the coefficients is zero.

From the first three equations it is seen that $\varphi$ is a function of

$$
l^{2}+m^{2}+n^{2}, \quad l^{\prime 2}+m^{\prime 2}+{n^{\prime}}^{2}, \quad l l^{\prime}+m m^{\prime}+n n^{\prime} ;
$$

calling these respectively $u, v$, and $w$, the last three equations become

where

$$
\begin{gathered}
2 l u^{\prime} \frac{\partial \varphi}{\partial u^{\prime}}+2 l^{\prime} v^{\prime} \frac{\partial \varphi}{\partial v^{\prime}}+\left(l+l^{\prime}\right) w^{\prime} \frac{\partial \varphi}{\partial w^{\prime}}=0 \\
2 m u^{\prime} \frac{\partial \varphi}{\partial u^{\prime}}+2 m^{\prime} v^{\prime} \frac{\partial \varphi}{\partial v^{\prime}}+\left(m+m^{\prime}\right) u^{\prime} \frac{\partial \varphi}{\partial w^{\prime}}=0 \\
2 n u^{\prime} \frac{\partial \varphi}{\partial u^{\prime}}+2 n^{\prime} v^{\prime} \frac{\partial \varphi}{\partial v^{\prime}}+\left(n+n^{\prime}\right) w^{\prime} \frac{\partial \varphi}{\partial w^{\prime}}=0
\end{gathered}
$$

$$
u^{\prime}=u+1, \quad v^{\prime}=v+1, \quad w^{\prime}=w+1,
$$

and, as should be the case, the determinant of the coefficients is zero. From any one of the three we have 


$$
\frac{d u^{\prime}}{u^{\prime}}+\frac{d v^{\prime}}{v^{\prime}}=\frac{d w^{\prime}}{w^{\prime}}
$$

that is, the solution $\varphi$ is of the form $\frac{w^{\prime 2}}{u^{\prime} v^{\prime}}$

or

$$
\frac{\left(l l^{\prime}+m m^{\prime}+n n^{\prime}+1\right)^{2}}{\left(l^{2}+m^{2}+n^{2}+1\right)\left(l^{2}+{m^{\prime 2}}^{2}+{n^{\prime 2}}^{2}+1\right)}=\cos ^{2} \theta
$$

and the invariant $\theta$ is defined to be the angle between the two linear manifoldnesses.

The fundamental notions of distance and direction are thus introduced by the invariants (4) and (7), the former relative to two elements of the space, the latter relative to two simplest manifoldnesses composed of a simply infinite number of these elements. All the derived notions of geometry may then be developed by as simple extensions of these two primary notions from three dimensions to four dimensions as occur when passing from the plane to ordinary space.

6. A curve in a flat space of four dimensions is said to have triple curvature when not every five consecutive points of it lie in a euclidean space of three dimensions. Such a curve possesses not only rectilinear tangents, and osculating planes, but also osculating spaces of three dimensions; by the latter is meant the limiting position of a secant three-dimensional space $\Sigma_{3}, M M^{\prime} M^{\prime \prime} M^{\prime \prime}$ as the three points $M^{\prime}, M^{\prime \prime}, M^{\prime \prime \prime}$ approach coincidence with $M$. At every point $M$ of the curve we have to consider a tetrarectangular tetraeder which has as edges the tangent, the principal normal, the principal binormal, and the trinormal. By the principal normal is meant the only one of the $\infty^{2}$ normals at every point which lies in the osculating plane; by the principal binormal is meant the only one of the $\infty^{1}$ binormals at every point which lies in the osculating space ; the trinormal is the only binormal perpendicular to the osculating space.

Taking the origin of the coördinate system at a point $M$ movable along the curve, assume the tangent as $x$-axis, the trinormal as $y$-axis, the principal binormal as z-axis, and the principal normal as $t$-axis. Let $\delta \varphi, \delta \psi, \delta \chi$ be the respective angles between two consecutive tangents, two adjacent principal binormals, and two adjacent trinormals, when the origin is shifted from $M$ to a neighboring point $M^{\prime}$ on the curve. The quadrangle of the direction cosines of the new axes referred to the old becomes 


\begin{tabular}{c|cccc} 
& $x$ & $y$ & $z$ & $t$ \\
\hline$x^{\prime}$ & 1 & 0 & 0 & $d \varphi$ \\
$y^{\prime}$ & 0 & 1 & $d \chi$ & 0 \\
$z^{\prime}$ & 0 & $-d \chi$ & 1 & $d \psi$ \\
$t^{\prime}$ & $-d \varphi$ & 0 & $-d \psi$ & 1
\end{tabular}

The limits of the ratios of $\delta \varphi, \delta \psi, \delta \chi$ to $M M^{\prime}$ as $M^{\prime}$ approaches $M$ measure the curvatures of the curve at $M$; the first limit is called the flexion, the second the torsion, and the third the curvature; or perhaps more simply, the first, second, and third curvatures. Then if we put

$$
d s=\rho d \varphi=\tau d x=r d \chi,
$$

the numbers $\rho, \tau, r$, the reciprocals of the curvatures, measure three lengths which are called the radii of the respective curvatures.

The array (11) shows that in order to discuss the curve in the domain of any of its points it is sufficient to know the functions $\varphi, \psi^{\prime}, \chi$; that is, it is sufficient to give $\rho, \tau, r$ as functions of $s$. Three equations, the so-called intrinsic equations

$$
f_{1}(\rho, \tau, r, s)=0, \quad f_{2}(\rho, \tau, r, s)=0, \quad f_{8}(\rho, \tau, r, 8)=0
$$

which determine the radii of curvature as functions of $s$, determine at the same time the forms of those arcs of the curve which lie between points at which the tangent may become indeterminate; they do not fix the position of these branches in space.

7. The method of the intrinsic analysis employed so successfully by Professor E. Cesàro in his Lezioni di geometria intrinseca and developed by him for curves and surfaces in spaces of many dimensions may be exhibited for curves of triple curvature in detail in the following manner.

Let $P$ be a point in space referred to $M$ as origin and generally movable with $M$; let its coördinates be $x(s), y(s)$ $z(s), t(s)$. Let $P^{\prime}$ be the point of the trajectory of $P$ corresponding to $M^{\prime}$; its coördinates referred to the axes at $M$ are $x+\delta x, y+\delta y, z+\delta z, t+\delta t$; its coördinates referred to the axes at $M^{\prime}$ are $x+d x, y+d y, z+d z, t+d t$; finally let $u, v$, $w, p$ be the coördinates of $M^{\prime}$ referred to $M$. Then projecting $M^{\prime} P^{\prime}$ on the axes at $M$, we have by virtue of the array (11) : 


$$
\begin{aligned}
& x+\delta x=u+x+d \psi-(t+d t) d \varphi, \\
& y+\delta y=v+y+d y-(z+d z) d \%, \\
& z+\delta z=w+z+d z+(y+d y) d \chi-(t+d t) d \psi, \\
& t+\delta t=p+t+d t+(x+d \alpha) d \varphi+(z+d z) d \psi .
\end{aligned}
$$

Excluding from further consideration here those curves for which it is not legitimate to assume that the limit of the ratio of the arc to the arc is unity, we have from the definition of the tangent as the limiting position of a secant

$$
£ \frac{u}{\delta s}=1, \quad £ \frac{v}{\delta s}=£ \frac{w}{\delta s}=£ \frac{p}{\delta s}=0,
$$

and the formulæ (12) give Cesàro's fundamental relations

$$
\begin{array}{ll}
\frac{\delta x}{d s}=\frac{d x}{d s}-\frac{t}{\rho}+1, & \frac{\delta y}{d s}=\frac{d y}{d s}-\frac{z}{r}, \\
\frac{\delta z}{d s}=\frac{d z}{d s}+\frac{y}{r}-\frac{t}{\tau}, & \frac{\delta t}{d s}=\frac{d t}{d s}+\frac{x}{\rho}+\frac{z}{\tau} .
\end{array}
$$

The formulæ (12) are also applicable to the cosines $\alpha, \beta$, $\gamma, \varepsilon$ that define any direction, provided that $u, v, w, p$ are zero, and the corresponding fundamental formulæ for this direction are found by replacing $x, y, z, t$ in (13) respectively by $\alpha, \beta, \gamma, \varepsilon$.

From (13) the necessary and sufficient conditions for the immobility of a point are

$$
\frac{d x}{d s}=\frac{t}{\rho}-1, \quad \frac{d y}{d s}=\frac{z}{r}, \quad \frac{d z}{d s}=\frac{t}{\tau}-\frac{y}{r}, \quad \frac{d t}{d s}=-\frac{x}{\delta}-\frac{\gamma}{\tau} .
$$

8. The above fundamental formulæ may be employed to show that every trio of intrinsic equations determines a curve uniquely, at least between convenient limits of $s$ in a range where the curvatures are finite and continuous functions of the arc. In fact there exists, by the theory of systems of linear differential equations, one and but one quatrain of functions $x(s), y(s), z(s), t(s)$ satisfying the simultaneous system (14), and reducing to $a, b, c, e$ for $s=0$. These functions $x, y, z, t$ are evidently the coördinates with regard to the tetraeder of origin $M$ of that point whose coördinates with regard to the tetraeder of origin $O$ are $a, b, c, e$.

As remarked above, the necessary and sufficient con- 
ditions for the invariability of the direction $(\alpha, \beta, \gamma, \varepsilon)$ are

$\frac{d x}{d s}=\frac{\varepsilon}{\rho}, \quad \frac{d \beta}{d s}=\frac{\gamma}{r}, \quad \frac{d \gamma}{d s}=-\frac{\beta}{r}+\frac{\varepsilon}{\tau}=, \quad \frac{d \varepsilon}{d t}=-\frac{\alpha}{\rho}-\frac{\gamma}{\tau} ;$

if $(\alpha, \beta, \gamma, \varepsilon$,$) and \left(\alpha^{\prime}, \beta^{\prime}, \gamma^{\prime}, \varepsilon^{\prime}\right)$ are any two solutions of this system it follows from the equations of the system that

$$
\begin{gathered}
\frac{d}{d s}\left(\alpha \alpha^{\prime}+\beta \beta^{\prime}+\gamma \gamma^{\prime}+\varepsilon \varepsilon^{\prime}\right)=0, \quad \frac{d}{d s}\left(\alpha^{2}+\beta^{2}+\gamma^{2}+\varepsilon^{2}\right)=0 \\
\frac{d}{d s}\left(\alpha^{\prime 2}+\beta^{\prime 2}+\gamma^{\prime 2}+\varepsilon^{\prime 2}\right)=0
\end{gathered}
$$

that is

$$
S \alpha \alpha^{\prime}, \quad S \alpha^{2}, \quad S \alpha^{\prime 2}
$$

are integrals of (15).

Hence if there be determined four systems of functions contained in the first of the following arrays

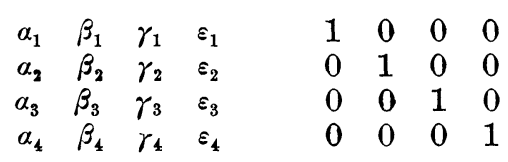

in such a manner that they satisfy (15) and take the corresponding values in the second array for $s=0$, we have

$$
S \alpha_{i} \alpha_{j}=0, \quad(i \neq j) ; \quad S \alpha_{i} \alpha_{j}=1, \quad(i=j) ;
$$

that is, the determinant represented by the first array is orthogonal. The elements of this determinant are the direction cosines of axes of origin $O$ with respect to those of origin $M$, since they give the directions of the former for $s=0$, and satisfy the conditions (15) for the invariability of those directions. Then in all cases

$$
\begin{array}{ll}
x=x_{0}+a \alpha_{1}+b \alpha_{2}+c \alpha_{3}+e \alpha_{4}, & y=y_{0}+a \beta_{1}+b \beta_{2}+c \beta_{3}+e \beta_{4}, \\
z=z_{0}+a \gamma_{1}+b \gamma_{2}+c \gamma_{3}+e \gamma_{4}, & t=t_{0}+a \varepsilon_{1}+b \varepsilon_{2}+c \varepsilon_{3}+e \varepsilon_{4} ;
\end{array}
$$

for each value of $s$ the constants $a, b, c, e$, can be determined in such manner that they represent the coördinates of $M$ with respect to axes of origin $O$; for this purpose it is sufficient to put $x, y, z, t$ equal to zero in the preceding equations and solve for $a, b, c, e$ :

$$
\begin{array}{ll}
a=-\left(\alpha_{1} x_{0}+\beta_{1} y_{0}+\gamma_{1} z_{0}+\varepsilon_{1} t_{0}\right), \quad b=-\left(\alpha_{2} x_{0}+\beta_{2} y_{0}+\gamma_{2} z_{0}+\varepsilon_{2} t_{0}\right), \\
c=-\left(\alpha_{3} x_{0}+\beta_{3} y_{0}+\gamma_{3} z_{0}+\varepsilon_{3} t_{0}\right), \quad e=-\left(\alpha_{4} x_{0}+\beta_{4} y_{0}+\gamma_{4} z_{0}+\varepsilon_{4} t_{0}\right) .
\end{array}
$$


Thus the curve defined by a trio of intrinsic equations is uniquely determined; by varying $s$ the coördinates $(a, b, c$, $e$ ) of all of its points with regard to a system of immovable axes are known.

9. It may be observed in conclusion that the whole theory of curves of triple curvature may be deduced by differentiation and elimination by means of the fundamental invariance conditions (14) and (15).

Differentiating (16) we have

$\frac{d a}{d s}=-S\left(x_{0} \frac{d x_{0}}{d s}+\alpha_{1} \frac{d x_{0}}{d s}\right)=\alpha_{1}, \quad \frac{d b}{d s}=\alpha_{2}, \quad \frac{d c}{d s}=\alpha_{3}, \quad \frac{d e}{d s}=\alpha_{4} ;$

also

$$
\begin{gathered}
\frac{d^{2} a}{d s^{2}}=\frac{\varepsilon_{1}}{\rho}, \cdots ; \\
\frac{d^{3} \alpha}{d s^{3}}=\varepsilon_{1} \frac{d}{d s} \frac{1}{\rho}-\frac{\alpha_{1}}{\rho^{2}}-\frac{\gamma_{1}}{\rho \tau}, \cdots .
\end{gathered}
$$

These formulæ lead to the following expressions for the curvatures as functions of the arc $s$ : for the first curvature, or flexion, which measures the rapidity with which the curve deviates from being a straight line,

$$
\frac{1}{\rho^{2}}=\left(\frac{d^{2} a}{d s^{2}}\right)^{2}+\left(\frac{d^{2} b}{d s^{2}}\right)^{2}+\left(\begin{array}{c}
d^{2} c \\
d s^{2}
\end{array}\right)^{2}+\left(\frac{d^{2} e}{d s^{2}}\right)^{2}
$$

for the second curvature, or torsion, which expresses the rapidity with which the curve deviates from being a plane curve,

$$
\frac{\frac{1}{\tau^{2}}=S\left(\frac{d^{2} a}{d s^{2}}\right)^{2} S\left(\frac{d^{3} a}{d s^{3}}\right)^{2}-\left\{S\left(\frac{d^{2} a}{d s^{2}}\right)^{2}\right\}^{3}-\left\{S \frac{d^{2} a}{d s^{2}} \frac{d^{3} a}{d s^{3}}\right\}}{\left\{S\left(\frac{d^{2} a}{d s^{2}}\right)^{2}\right\}^{2}} ;
$$

finally for the third curvature, which measures the rapidity of deviation from a curve of double curvature,

$$
\frac{1}{r^{2}}=\left(\frac{d A}{d s}\right)^{2}+\left(\frac{d B}{d s}\right)^{2}+\left(\frac{d C}{d s}\right)^{2}+\left(\frac{d E}{d s}\right)^{2}
$$

where $A=A^{\prime} / D, B=B^{\prime} / D, C=C^{\prime} / D, E=E^{\prime} / D$, $D^{2}=A^{\prime 2}+B^{\prime 2}+C^{\prime 2}+E^{\prime 2}$ 
$A^{\prime}, B^{\prime}, C^{\prime}, E^{\prime}$ being the minors corresponding to $a, b, c, e$ in the Wronskian

$$
W=\left|a, \quad \frac{d b}{d s}, \quad \frac{d^{2} e}{d s^{2}}, \quad \frac{d^{3} e}{d s^{3}}\right| \text {. }
$$

The above values for the curvatures are derived directly by Pirondini in Battaglini's Journal for 1890.

Princeton, New Jersey.

\section{NOTES.}

The closing (October) number of Volume I of the Transactions of the American Mathematical Society contains the following papers: "On surfaces enveloped by spheres belonging to a linear spherical complex," by P. F. Sмгтн ; " On certain relations among the theta constants," by J. I. Hutchinson; "On groups which have the same group of isomorphisms," by G. A. Mrller ; "Die Hesse'sche und die Cayley'sche Curve," by P. Gordan ; "Application of a method of D'Alembert to the proof of Sturm's theorem of comparison," by M. BôcHer ; "Two plane movements generating quartic scrolls," by E. M. BLAKE ; "The invariant theory of the inversion group: geometry upon a quadric surface," by E. KASNER ; "A simple proof of the fundamental Cauchy-Goursat theorem." by E. H. Moore ; "Notes and errata : Volume I.'

THE mathematical section of the British association for the advancement of science held its meeting at Bradford, September 10, 1900, with Major P. A. MacMahon, F.R.S. as presiding officer, and Mr. E. T. Whittaker, secretary. The following papers were read:

(1) Miss F. Hardcastle : Report (preliminary) on the present state of the theory of point groups.

(2) Major P. A. MacMahon : "A property of the characteristic symbolic determinant of any $n$ quantics in $n$ variables.'"

(3) Professor Cyparissos Stephanos: "Sur les relations entre la géométrie projective et la mécanique."

(4) Mr. H. S. Carslaw : "The use of multiple space in applied mathematics."'

(5) Major P. A. MacMaHoN": "The asyzygetic and perpetuant covariants of systems of binary quantics." 